\title{
Proteomics and metabolomics of raw rhubarb and wine-processed rhubarb in the treatment of rats with intracerebral hemorrhage
}

\author{
Kun Wang ${ }^{1}$, Dongsheng Guan ${ }^{2}$, Xu Zhao ${ }^{1}$, Dongge Qiao ${ }^{3}$, Yanhua Yang ${ }^{1}$, Yinglin Cui ${ }^{2}$ \\ ${ }^{1}$ Department of Pharmacy, Henan Province Hospital of TCM (The Second Clinical Medical College, Henan University of Traditional Chinese \\ Medicine), Zhengzhou, China; ${ }^{2}$ Department of Encephalopathy, Henan Province Hospital of TCM (The Second Clinical Medical College, Henan \\ University of Traditional Chinese Medicine), Zhengzhou, China; ${ }^{3}$ Nursing Department, Henan Province Hospital of TCM (The Second Clinical \\ Medical College, Henan University of Traditional Chinese Medicine), Zhengzhou, China \\ Contributions: (I) Conception and design: Y Cui, K Wang, D Qiao; (II) Administrative support: Y Cui; (III) Provision of study materials or patients: \\ K Wang, D Guan; (IV) Collection and assembly of data: Y Yang; (V) Data analysis and interpretation: K Wang, D Qiao; (VI) Manuscript writing: All \\ authors; (VII) Final approval of manuscript: All authors. \\ Correspondence to: Yinglin Cui. NO. 6 Dongfeng Road, Jinshui District, Zhengzhou 450002, China. Email: hnszyy001@126.com.
}

Background: To explore the mechanisms of raw rhubarb and wine-processed rhubarb treatment in a rat model of intracerebral hemorrhage (ICH).

Methods: After adapting to their environment, 30 male Wistar rats were divided into 5 treatment groups: blank control group (CK) (normal saline), sham operation group (SICH) (normal saline), ICH model group (ICH) (normal saline), ICH + raw rhubarb enema group (RO) (raw rhubarb $3.60 \mathrm{~g} / \mathrm{kg}$ ), and ICH + wineprocessed rhubarb enema group (WRO) (wine-processed rhubarb $3.60 \mathrm{~g} / \mathrm{kg}$ ). The rhubarb enema (once a day) was given since 3 days before ICH treatment, and was consistently given until 12 hours, 24 hours, 3 days, and 7 days post operation. Serum oxidative stress and inflammatory markers were detected by ELISA, and differences between raw rhubarb and wine-processed rhubarb treatment in ICH rats were screened by proteomics and metabolomics.

Results: Both rhubarb treatments effectively improved oxidative stress and inflammatory responses. After $\mathrm{ICH}$, the proteins and metabolites in the brain tissue were significantly altered. Compared with raw rhubarb, wine-processed rhubarb had a better protective effect by dysregulating amino acid metabolism in the ICH model.

Conclusions: Our study provides a basis for further research into the mechanisms of rhubarb treatment from different processing technologies, and promotes the application of rhubarb as a therapeutic approach to ICH.

Keywords: Proteomics; metabolomics; intracerebral hemorrhage (ICH); raw rhubarb; wine-processed rhubarb

Submitted Oct 28, 2020. Accepted for publication Dec 22, 2020.

doi: 10.21037/atm-20-7831

View this article at: http://dx.doi.org/10.21037/atm-20-7831

\section{Introduction}

Intracerebral hemorrhage (ICH) refers to hemorrhage caused by the rupture of blood vessels in non-traumatic brain parenchyma. It involves a variety of injury mechanisms, including oxidative stress responses, neuroexcitotoxicity, neuronal apoptosis, and inflammatory responses, which can lead to changes in metabolites such as lipids, nucleic acids, and glucose $(1,2)$. Oxidative stress occurs first during hemorrhage, and the failure of sodium potassium ion pumps results in the loss of membrane ion gradient and nerve depolarization, leading to brain edema (3). In addition, the activation of inflammatory responses 
mediated by cytokines is also an important factor in the damage to the blood-brain barrier (4).

Rhubarb, especially wine-processed rhubarb, can alleviate the inflammatory responses of the brain and protect the blood-brain barrier after cerebral hemorrhage (5). Raw rhubarb has a strong purgative effect, which is alleviated after processing with Chinese rice wine (6). Chinese rice wine is thought to increase the dissolution of active compounds, and promote blood circulation. It was found that wine-processed rhubarb can significantly change the distribution of aloe-emodin, rhein, and emodin in rats (6). However, the mechanisms and targets of rhubarb in ICH remain unknown.

Proteomics is the large-scale analysis of proteins, including post-translational modifications and protein interactions, so as to reveal the function of proteins. Proteomics has been used in the screening of vaccines, clinical drug development, the prediction of prognosis, and to guide treatment approaches (7). Metabolomics is the qualitative and quantitative analysis of all low molecular weight metabolites in the biological system at a specific time and under specific conditions, and describes the responses of endogenous metabolites to internal and external changes. Metabolomics has been widely used in toxicology, pharmacology, nutrition, and disease amongst other research fields (8). In order to further explore the mechanisms and targets of rhubarb and wine-processed rhubarb on ICH, we performed proteomics and metabolomics analyses in a rat model of ICH treated with raw rhubarb or wine-processed rhubarb.

We present the following article in accordance with the ARRIVE reporting checklist (available at http://dx.doi. org/10.21037/atm-20-7831).

\section{Methods}

\section{Drug preparation}

An appropriate amount of Chinese rice wine was added to raw rhubarb until the slices were thoroughly moistened. The rhubarb slices were then stir-fried to dry on a gentle fire to produce wine-processed rhubarb.

A total of $80 \mathrm{~g}$ of rhubarb was weighed, refluxed with $75 \%$ ethanol for $1 \mathrm{~h}$ and extracted twice. Decompression was performed to obtain a crude drug concentration of $0.9 \mathrm{~g} / \mathrm{mL}$ of the rhubarb extract. The preparation method of wine-processed rhubarb extract was the same as above.

\section{Grouping design, drug treatments and model preparation}

Experiments in our research were performed under a project license granted by the Institutional Animal Care and Use committee of Henan Province Hospital of TCM (Second Affiliated Hospital of Henan University of Traditional Chinese Medicine), in compliance with national guidelines for the care and use of animals.

Eight-week-old SPF male adult Wistar rats (250-300 g, $\mathrm{n}=30$ ) were obtained from Beijing Vital River Laboratory Animal Technology Co. Ltd. (Beijing, China). Rats were randomly divided into 5 groups: blank control group (CK), sham operation group (SICH), ICH model group (ICH), $\mathrm{ICH}+$ raw rhubarb enema group (RO), ICH + wineprocessed rhubarb enema group (WRO), with 6 rats in each group, and kept in an air-conditioned room under the constant room temperature $\left(23 \pm 2{ }^{\circ} \mathrm{C}\right)$, relative humidity level $(50 \% \pm 15 \%)$, and lighting (12 h light/dark cycle) with free access to food and water. Rats were anesthetized with $4 \%$ chloral hydrate $(8 \mathrm{~mL} / \mathrm{kg})$ via intraperitoneal injection, and placed on a stereotaxic apparatus with craniotomy performed under sterile conditions. Type VII collagenase $(0.5 \mu \mathrm{L})$ was extracted from the boreholes $0.2 \mathrm{~mm}$ in front and $3 \mathrm{~mm}$ outside bregma with a microsyringe and injected into the caudate nucleus. Collagenase was injected within 3 minutes, the needle remained in place for 10 minutes, then retracted, and the skin incision was sutured. The sham operation group was injected with sterile saline instead of collagenase. After the operation, the rats were kept warm until they completely recovered from the anesthesia, and then Longa's scoring method was used to preliminarily verify whether there were neural defects.

The CK group (normal saline), ICH group (normal saline), SICH group (normal saline), RO group (raw rhubarb $3.60 \mathrm{~g} / \mathrm{kg}$ ), and WRO group (wine-processed rhubarb $3.60 \mathrm{~g} / \mathrm{kg}$ ) were given enemas for 3 days (once a day). The rhubarb enema (once a day) was given since 3 days before ICH treatment, and was consistently given until 12 hours, 24 hours, 3 days, and 7 days post operation. Rats were euthanatized after blood collection from the abdominal vessel, and the brain tissue of the bleeding side was extracted.

\section{Detection of oxidative stress and inflammatory markers in serum}

Abdominal artery serum $(2 \mathrm{~mL})$ from the rats was taken 
before euthanasia. The glutathione (GSH) level in serum was detected using ELISA. The optical density (OD value) was measured at the wavelength of $450 \mathrm{~nm}$.

\section{Metabolomics analysis}

Chromatography was performed on the Ultimate 3000 system (Thermo Fisher Scientific, USA). The following quantitative analysis was performed on quadrupole-orbitrap mass spectrometer equipped with an electrospray ionization (ESI) interface and an orbitrap mass analyzer (Thermo Fisher Scientific, USA).

\section{Metabolite extraction}

Animal tissue $(100 \mathrm{mg})$ was dissolved in $1,000 \mu \mathrm{L}$ of $80 \%$ methanol $\left(\mathrm{V} / \mathrm{V},-20^{\circ} \mathrm{C}\right)$, then grinded and disrupted with an ultrasonic homogenizer. The supernatants were centrifuged at $14,000 \mathrm{rpm}$ for $10 \mathrm{~min}$ at $4{ }^{\circ} \mathrm{C}$, and $800 \mu \mathrm{L}$ was concentrated by vacuum concentration. The sample was re-dissolved with $50 \%$ methanol containing $400 \mu \mathrm{L}$ 2-chlorophenylalanine (4 ppm). The samples were filtered with a $0.22 \mu \mathrm{m}$ filter membrane and obtained for LC-MS detection. The quality control (QC) samples, a mixture of $20 \mu \mathrm{L}$ from each sample, were prepared using the same procedures described above.

\section{Liquid chromatography}

Chromatographic separation was accomplished in Ultimate 3000 system equipped (Thermo Scientific) with an ACQUITY UPLC ${ }^{\circledR}$ HSS T3 $(150 \times 2.1 \mathrm{~mm}, 1.8 \mu \mathrm{m}$, Waters) column maintained at $40{ }^{\circ} \mathrm{C}$. The temperature of the autosampler was $8^{\circ} \mathrm{C}$. Gradient elution of analytes was carried out with $0.1 \%$ formic acid in water (I) and $0.1 \%$ formic acid in acetonitrile (II) or $5 \mathrm{mM}$ ammonium formate in water (III) and acetonitrile (IV) at a flow rate of $0.25 \mathrm{~mL} / \mathrm{min}$. Two $\mu \mathrm{L}$ of each sample was injected after equilibration. Increasing linear gradient of solvent $\mathrm{B}(\mathrm{V} / \mathrm{V})$ : 0-1 $\mathrm{min}, 2 \% \mathrm{~B} / \mathrm{D} ; 1-9 \mathrm{~min}, 2-50 \% \mathrm{~B} / \mathrm{D} ; 9-12 \mathrm{~min}$, $50-98 \% \mathrm{~B} / \mathrm{D} ; 12-13.5 \mathrm{~min}, 98 \% \mathrm{~B} / \mathrm{D} ; 13.5-14 \mathrm{~min}$, 98-2\% B/D; $14-17$ min, 2\% B/D.

\section{Mass spectrometry conditions}

The mass spectrometry conditions were optimized as follows: sheath gas flow rate, 45 arb; auxiliary gas flow rate, $15 \mathrm{arb}$; spray voltage of positive ions, $3.8 \mathrm{kV}$; spray voltage of negative ions, $-2.5 \mathrm{kV}$; capillary temperature, $325{ }^{\circ} \mathrm{C}$. Compounds detecting is completed using a full MS scan, which covers a mass range of $\mathrm{m} / \mathrm{z} 80-1,000$. Both positive and negative ions were scanned in a mode of fast switching between positive and negative ions under the condition of resolution of 70,000 full width half maximum (FWHM). Data dependent acquisition (DDA) were performed with a Higher energy C-trap dissociation (HCD) scan. The normalized collision energy was $30 \mathrm{eV}$. Dynamic exclusion was implemented to remove some unnecessary information in the MS/MS spectra.

\section{Metabolite identification, differential metabolite screening, and functional analysis}

Human Metabolome Database (HMDB) (http://www.hmdb.ca), Metlin (http://metlin.scripps.edu), MassBank (http://www. massbank.jp/), Lipid Maps (http://www.lipidmaps.org), and mzclound (https://www.mzcloud.org) were used to identify metabolites. The differential compounds were screened by $\mathrm{P}<0.05$ and variable importance in the projection (VIP) $\geq 1$ from the OPLS-DA model.

\section{Kyoto Encyclopedia of Genes and Genomes (KEGG) patbway enrichment of metabolites}

Pathway analysis was performed using the MetaboAnalyst $R$ package to observe the role of differential endogenous metabolites in possible signaling pathways.

\section{Proteomics analysis}

\section{Protein extraction}

The brain tissue (the same hemisphere of the hemorrhage area) was lysed in $1.5 \mathrm{~mL}$ lysis buffer (7 M urea, 4\% SDS, $30 \mathrm{mM}$ HEPES, $2 \mathrm{mM}$ EDTA, $10 \mathrm{mM}$ DTT, containing $1 \times$ proteinase inhibitor cocktail and $1 \mathrm{mM}$ PMSF) and homogenized with ultrasound for $10 \mathrm{~min}$ on ice. The lysates were centrifuged at $20,000 \mathrm{~g}$ for 30 minutes at $4{ }^{\circ} \mathrm{C}$, and the supernatants were collected and quantified with BCA Kit (Thermo fisher scientific, USA). One hundred $\mu \mathrm{g}$ of the protein samples was reduced by $10 \mathrm{mM}$ DTT at $56^{\circ} \mathrm{C}$ for $1 \mathrm{~h}$ and alkylated by $55 \mathrm{mM}$ IAA in the dark at room temperature for $1 \mathrm{~h}$. The protein samples were precipitated with pre-cooled acetone (4 times the sample volume) at $-20{ }^{\circ} \mathrm{C}$ for more than $3 \mathrm{~h}$, and pelleted by centrifugation at $20,000 \mathrm{~g}$ for $30 \mathrm{~min}$ at $4{ }^{\circ} \mathrm{C}$. Acetone/ ethanol solution $(\mathrm{V} / \mathrm{V}=1: 1)$ was added $(1 \mathrm{~mL})$ to precipitate 
at $-20{ }^{\circ} \mathrm{C}$ degrees for more than 3 hours. The precipitate was centrifuged at $4^{\circ} \mathrm{C}$ at $20,000 \mathrm{~g}$ for $30 \mathrm{~min}$. This step was repeated twice. Ammonium bicarbonate (ABC) was added $(100 \mathrm{mM})$ to all samples until the volume was $100 \mu \mathrm{L}$. Trypsin was added to the samples at a mass ratio of $1: 100$ (enzyme:protein), and the proteins were lysed at $37^{\circ} \mathrm{C}$ for $4 \mathrm{~h}$. The samples were then supplemented with an extra $1.0 \mu \mathrm{g}$ Trypsin at $37^{\circ} \mathrm{C}$ for $12 \mathrm{~h}$. Finally, the protein samples were pelleted with centrifugation at 5,000 $\mathrm{g}$ and desalted with the C18 columns (Thermo fisher scientific, USA). The elution was freeze-dried to remove acetonitrile and supplied for LS-MS/MS.

\section{Proteomics conditions}

The spray voltage of the ion source was $2.2 \mathrm{kV}$. The mass spectrometer heated the capillary tube at $320{ }^{\circ} \mathrm{C}$, and the collection was automatically switched between MS and MS/MS in a data-dependent mode. Full scan MS used Orbitrap to perform a primary scan with a range of $\mathrm{m} / \mathrm{z}$ $350-1,550$ and a resolution of $120,000(\mathrm{~m} / \mathrm{z} 200)$. Ion biggest introduction of $50 \mathrm{~ms}$, automatic gain control (AGC) set to $5 \times 10^{5}$, then used high-energy collisions in 3 second dissociations (who energy C-trap dissociation, HCD) to conform to the cascade (MS/MS) fragmentation conditions of ion fragmentation and orbitrap scanning, scanning resolution was set to 15,000 . The scanning range was automatically controlled according to the mass/charge ratio of the parent ions. The lowest scanning range was fixed at $110 \mathrm{~m} / \mathrm{z}$ and the highest was up to 2,000. The minimum ion strength value of MS/MS was set to 50,000. When $\mathrm{MS} / \mathrm{MS}$, the maximum ion introduction time was $100 \mathrm{~ms}$, the AGC control was set to $2.0 \times 10^{5}$, and the ion selection window was set to 1.6 Daltons. For ions with 2, 3 and 4 charge Numbers, MS/MS collection was carried out, and the dynamic exclusion was set as MS/MS once for each ion within 10 seconds, after which the collision energy of $30 \%$ was eliminated for 30 seconds.

\section{Qualitative and quantitative analysis of proteins}

Raw files of mass spectrometry data were analyzed using MaxQuant (version 1.5.8.3). MaxQuant software used the iBAQ algorithm for quantitative analysis according to the search results. Protein quantification requires that each protein contain at least 2 unique peptides, and the quantitative protein ratio was weighted and normalized at the median ratio.

\section{Western blotting}

Total proteins of the brain tissues were extracted by RIPA lysis buffer (Beyotime, China) and concentrated by BCA protein Assay Kit (Thermo Fisher Scientific, USA). The total proteins were separated by SDS-PAGE and transferred onto a PVDF membrane (Millipore, USA). The PVDF membrane was blocked with $5 \%$ slim milk and incubated with specific antibodies (anti-EEF1E1, ab31543, Abcam; anti-SAR1A, 22291-1-AP, Proteintech; anti-PLXNB1, 23795-1-AP, Proteintech; anti-DAG1, ab234587, Abcam; anti-OCIAD2, ab118565, Abcam). The PVDF membranes were washes with TBS buffer containing $0.05 \%$ Tween-20 (TBST) and incubated with the secondary antibodies and washed in TBST. Finally, proteins were visualized by enhanced chemiluminescence (ECL, Thermo Fisher Scientific, USA), and the relative expression levels of the proteins were analyzed by Image J software.

\section{Statistical analyses}

Data was expressed as mean \pm SD. ANOVA was used to compare multiple groups, and $t$-test was used to compare two groups. $\mathrm{P}<0.05$ was considered statistically significant.

\section{Results}

\section{Wine-processed rhubarb increases the expression of GSH in ICH rats}

There was no significant difference in GSH between the sham group and the blank group (Table 1). Compared with the sham group, the GSH content in the brain tissue of rats in the ICH model group was significantly reduced $(\mathrm{P}<0.01)$. Compared with the ICH model group, GSH content in the brain tissue of rats in the raw rhubarb group increased at $24 \mathrm{~h}(\mathrm{P}<0.05)$, and also significantly increased at 3 and $7 \mathrm{~d}(\mathrm{P}<0.01)$, while GSH levels in the brain tissue of rats in the wine-processed rhubarb group increased significantly at 24 h, $3 \mathrm{~d}$, and $7 \mathrm{~d}(\mathrm{P}<0.01)$. Compared with the raw rhubarb group, the GSH contents in the brain tissue of rats in the wine-processed rhubarb group also increased significantly.

\section{Wine-processed rhubarb can reduce the expression of inflammatory factors in $\mathrm{ICH}$ rats}

There was no significant difference in the levels of 
Table 1 Effects of raw rhubarb and wine-processed rhubarb on GSH content in ICH rats $(\bar{x} \pm \mathbf{s}, \mathrm{n}=6)$

\begin{tabular}{lcccc}
\hline & $12 \mathrm{~h}\left(\mu \mathrm{mol} \cdot \mathrm{L}^{-1}\right)$ & $24 \mathrm{~h}\left(\mu \mathrm{mol} \cdot \mathrm{L}^{-1}\right)$ & $3 \mathrm{~d}\left(\mu \mathrm{mol} \cdot \mathrm{L}^{-1}\right)$ & $7 \mathrm{~d}\left(\mu \mathrm{mol} \cdot \mathrm{L}^{-1}\right)$ \\
\hline $\mathrm{CK}$ & $152.51 \pm 8.71$ & $151.56 \pm 4.73$ & $154.50 \pm 14.71$ & $159.05 \pm 10.13$ \\
$\mathrm{SICH}$ & $162.18 \pm 6.10^{\star \star}$ & $161.80 \pm 12.38^{\star *}$ & $146.31 \pm 15.61^{\star \star}$ & $168.02 \pm 14.72^{\star \star}$ \\
$\mathrm{ICH}$ & $116.70 \pm 7.38$ & $73.35 \pm 12.56$ & $82.10 \pm 7.05$ & $72.18 \pm 7.52$ \\
$\mathrm{RO}$ & $130.77 \pm 3.43^{*}$ & $108.43 \pm 13.28^{* *}$ & $114.72 \pm 9.86^{\star *}$ & $104.52 \pm 9.84^{\star \star}$ \\
WRO & $120.36 \pm 10.98$ & $97.51 \pm 10.93^{*}$ & $111.92 \pm 3.58^{\star *}$ & $102.23 \pm 12.10^{\star *}$ \\
\hline
\end{tabular}

*, $\mathrm{P}<0.05$; ** $\mathrm{P}<0.01$. $\mathrm{CK}$, blank control group; $\mathrm{SICH}$, sham operation group; ICH, ICH model group; $\mathrm{RO}$, ICH + raw rhubarb enema group; $\mathrm{WRO}, \mathrm{ICH}+$ wine-processed rhubarb enema group.

Table 2 Effects of raw rhubarb and wine-processed rhubarb on TNF- $\alpha$ content in ICH rats $(\bar{x} \pm \mathrm{s}, \mathrm{n}=6)$

\begin{tabular}{lccrr}
\hline & $12 \mathrm{~h}(\mathrm{pg} / \mathrm{mL})$ & $24 \mathrm{~h}(\mathrm{pg} / \mathrm{mL})$ & $3 \mathrm{~d}(\mathrm{pg} / \mathrm{mL})$ & $7 \mathrm{~d}(\mathrm{pg} / \mathrm{mL})$ \\
\hline $\mathrm{CK}$ & $126.00 \pm 25.859$ & $126.00 \pm 25.859$ & $126.00 \pm 25.859$ & $126.00 \pm 25.859$ \\
$\mathrm{SICH}$ & $162.50 \pm 7.778^{\star *}$ & $157.00 \pm 25.456^{\star *}$ & $116.50 \pm 31.820^{\star *}$ & $166.50 \pm 20.506^{\star \star}$ \\
$\mathrm{ICH}$ & $317.60 \pm 35.997$ & $405.80 \pm 34.867$ & $531.60 \pm 55.788$ & $497.00 \pm 30.290$ \\
RO & $130.77 \pm 3.43^{*}$ & $108.43 \pm 13.28^{\star \star}$ & $114.72 \pm 9.86^{\star *}$ & $104.52 \pm 9.84^{\star *}$ \\
WRO & $120.36 \pm 10.98$ & $97.51 \pm 10.93^{*}$ & $111.92 \pm 3.58^{\star \star}$ & $102.23 \pm 12.10^{\star \star}$ \\
\hline
\end{tabular}

*, $\mathrm{P}<0.05$; ** $\mathrm{P}<0.01$. CK, blank control group; $\mathrm{SICH}$, sham operation group; ICH, ICH model group; $\mathrm{RO}$, ICH + raw rhubarb enema group; $\mathrm{WRO}, \mathrm{ICH}+$ wine-processed rhubarb enema group.

inflammatory factors between the sham group and the blank group. Compared with the sham group, TNF- $\alpha$ levels in $\mathrm{ICH}$ model group increased significantly at the 4 time points $(\mathrm{P}<0.01)$. Compared with the ICH model group, TNF- $\alpha$ was significantly reduced in the raw rhubarb group at $24 \mathrm{~h}, 3 \mathrm{~d}$, and $7 \mathrm{~d}(\mathrm{P}<0.01)$, with a decreasing trend at $12 \mathrm{~h}$ that did not reach statistical significance. TNF-a content in the wine-processed rhubarb group significantly decreased at $12 \mathrm{~h}(\mathrm{P}<0.05), 24 \mathrm{~h}, 3 \mathrm{~d}$, and $7 \mathrm{~d}(\mathrm{P}<0.01)$. Compared with the raw rhubarb group, the content of TNF- $\alpha$ in the ICH rats in the wine-processed rhubarb group was significantly reduced. The data are shown in Table 2.

There was no significant difference in IL-6 levels between the sham group and the blank group. Compared with the sham group, IL-6 levels in the ICH model group increased significantly at the 4 time points $(\mathrm{P}<0.01)$. Compared with the ICH model group, the content of IL-6 in the raw rhubarb group significantly decreased at 12 and $24 \mathrm{~h}(\mathrm{P}<0.05)$, and also at 3 and $7 \mathrm{~d}(\mathrm{P}<0.01)$. The content of IL-6 in the wine-processed rhubarb group significantly decreased at 12 and $24 \mathrm{~h}(\mathrm{P}<0.05)$, and also at 3 and $7 \mathrm{~d}(\mathrm{P}<0.01)$. Compared with the raw rhubarb group, the content of IL-6 in ICH rats in the wine-processed rhubarb group was significantly reduced. The data are shown in the Table 3.

\section{Differentially expressed proteins between raw rbubarb and wine-processed rhubarb}

In order to identify the targets of the response to raw rhubarb or wine-processed rhubarb treatment, we quantified the proteins secreted by brain tissue after treatment with the 2 kinds of rhubarb. As shown in Figure 1, there were 87 differentially expressed proteins between the raw rhubarb and wine-processed rhubarb groups, among which 5 proteins (eef1e1, sar1a, plxnb1, DAG1, ociad2) were significantly dysregulated, suggesting that these proteins may be the key proteins responsible for the different effects between raw rhubarb and wine-processed rhubarb. Western blotting further confirmed the expression of these proteins (see Figure S1). The Venn diagram also showed that there were 221 specific proteins expressed in raw rhubarb, compared with wine-processed rhubarb group (up 102, down 119), while 123 specific proteins expressed in wineprocessed rhubarb compared with raw rhubarb group (up 59, down 64) The different effects of raw rhubarb and wine- 
Table 3 Effects of raw rhubarb and wine-processed rhubarb on IL- 6 content in ICH rats $(\bar{x} \pm \mathrm{s}, \mathrm{n}=6)$

\begin{tabular}{|c|c|c|c|c|}
\hline & $12 \mathrm{~h}(\mathrm{pg} / \mathrm{mL})$ & $24 \mathrm{~h}(\mathrm{pg} / \mathrm{mL})$ & $3 \mathrm{~d}(\mathrm{pg} / \mathrm{mL}))$ & $7 \mathrm{~d}(\mathrm{pg} / \mathrm{mL})$ \\
\hline $\mathrm{SICH}$ & $71.50 \pm 12.02^{\star \star}$ & $74.00 \pm 15.56^{\star \star}$ & $82.50 \pm 10.61^{\text {** }}$ & $81.50 \pm 20.51^{\star *}$ \\
\hline $\mathrm{ICH}$ & $155.40 \pm 17.59$ & $200.80 \pm 20.38$ & $237.80 \pm 20.66$ & $229.20 \pm 20.95$ \\
\hline RO & $134.00 \pm 19.42^{*}$ & $169.00 \pm 14.88^{*}$ & $184.00 \pm 20.43^{\star *}$ & $177.40 \pm 23.73^{\star \star}$ \\
\hline
\end{tabular}

*, $\mathrm{P}<0.05$; ${ }^{*}, \mathrm{P}<0.01$. CK, blank control group; $\mathrm{SICH}$, sham operation group; ICH, ICH model group; $\mathrm{RO}$, ICH + raw rhubarb enema group; $\mathrm{WRO}, \mathrm{ICH}+$ wine-processed rhubarb enema group.

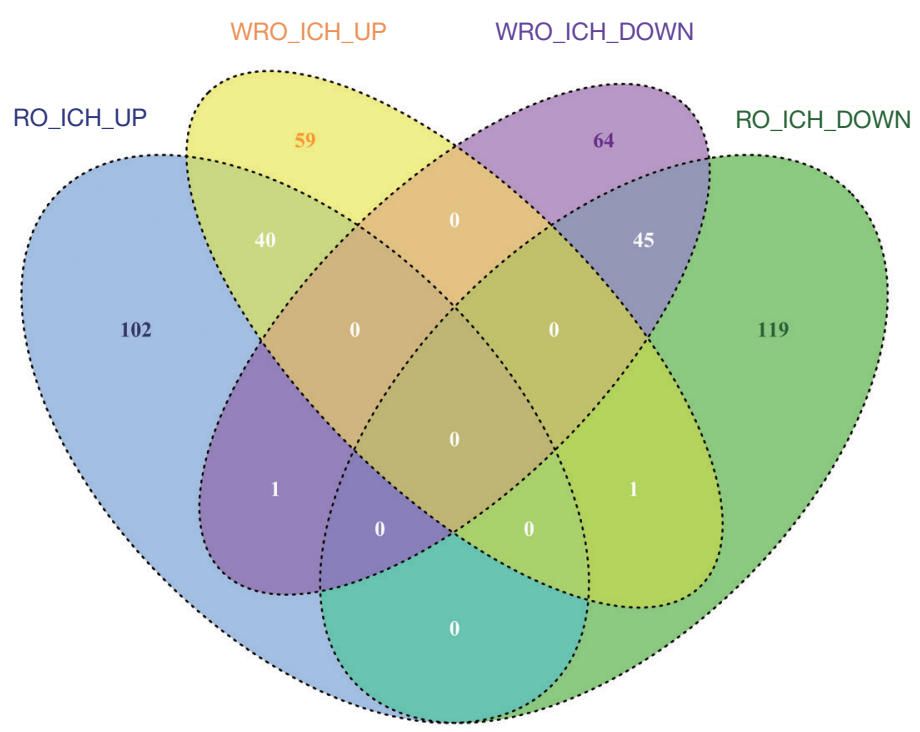

Figure 1 Comparative analysis of different proteins between the raw rhubarb and the wine-processed rhubarb treatment groups.

processed rhubarb on the prevention and treatment of ICH were most likely controlled by these genes. Therefore, these differentially expressed proteins were further analyzed.

\section{KEGG enrichment analysis of proteins in the raw rhubarb and wine-processed rhubarb treatment groups}

We further analyzed the KEGG enrichment of differentially expressed proteins between raw and wineprocessed rhubarb treatment. The differentially expressed proteins of raw rhubarb treatment were mainly enriched in carbon metabolism, citrate cycle (TCA cycle), endocytosis, glycolysis/gluconeogenesis, pyruvate metabolism, aldosterone-regulated sodium reabsorption, AMPK signaling, PPAR signaling, insulin signaling, oxidative phosphorylation, and circadian rhythm, as shown in
Figure $2 A$. Differentially expressed proteins of wine-processed rhubarb treatment were mainly enriched in valine, leucine, and isoleucine degradation, glycine, serine, and threonine metabolism, amino acid biosynthesis, phospholipase D signaling pathway, arginine and proline metabolism, alanate metabolism, snare interactions in vesicular transport, phosphatidylinositol signaling system, axon guidance, cholinergic synapse, leukocyte migration across the endothelium, and adhesion spot, as shown in Figure $2 B$.

\section{Metabolomics analysis}

\section{Principal component analysis (PCA)}

PCA was carried out for ICH, RO, and WRO. The PCA scores (Figure 3) obtained under the positive and negative ion modes showed that each treatment group had significant differences. 
A

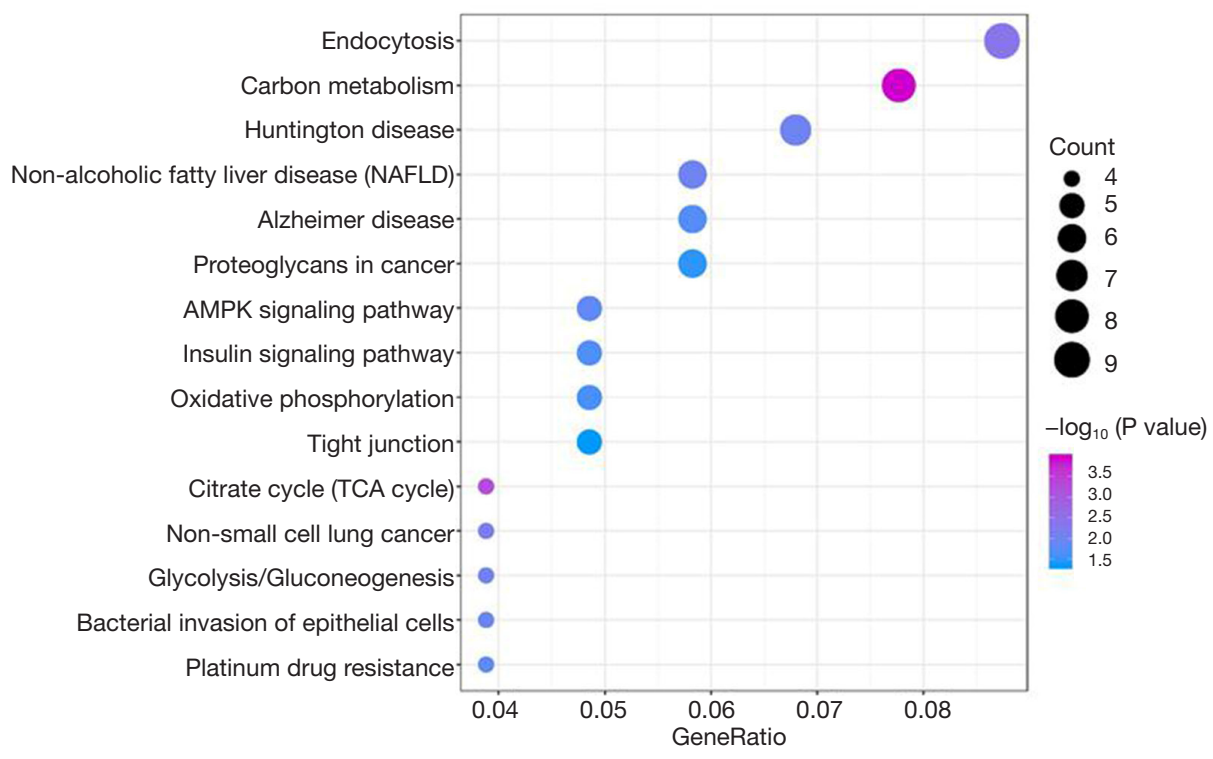

B

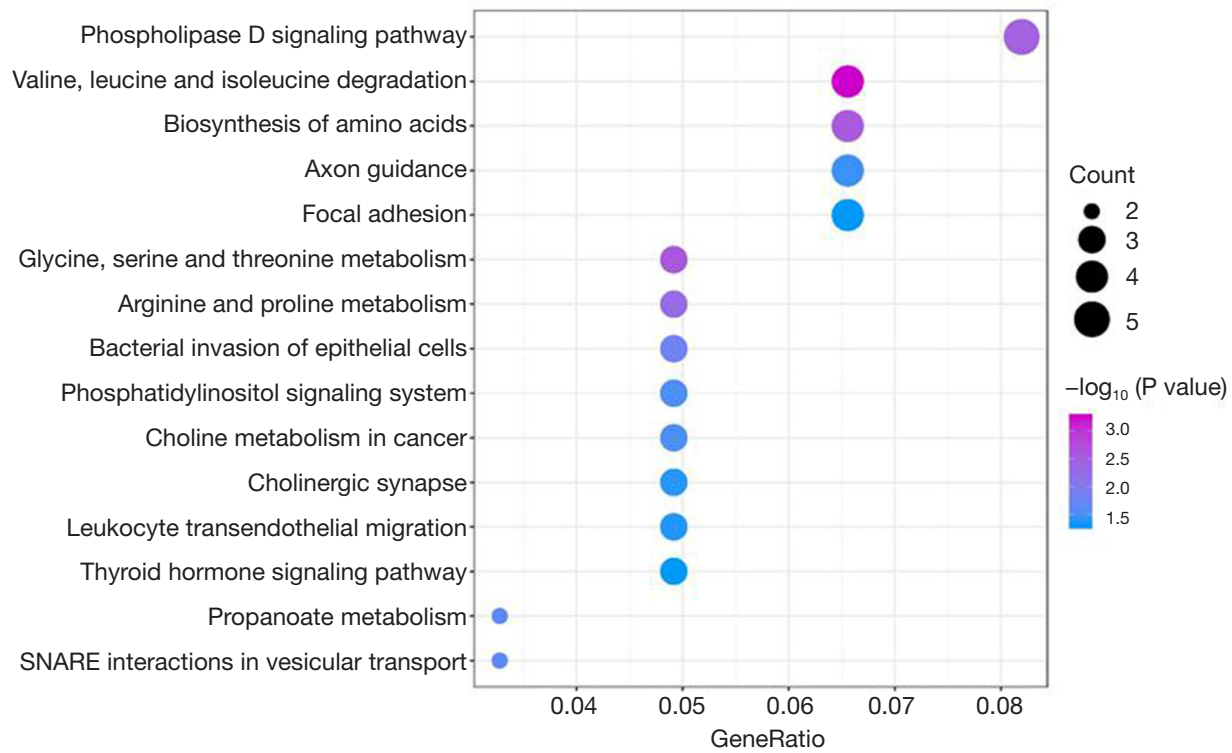

Figure 2 Kyoto Encyclopedia of Genes and Genomes (KEGG) enrichment analysis of proteins in the raw rhubarb and wine-processed rhubarb treatment groups. (A) KEGG enrichment analysis of proteins in the raw rhubarb treatment group; (B) KEGG enrichment analysis of proteins in the wine-processed rhubarb treatment group. The abscissa generatio represents the multiple of the total proportion of differentially expressed proteins in the Gene Ontology (GO) or KEGG relative to the proportion of proteins identified in the classification. The vertical coordinate represents GO or KEGG term description, and the bubble represents the number of differential proteins in the GO or KEGG classification; Fisher's exact test $\mathrm{P}$ value: the $\mathrm{P}$ value of the enrichment test obtained by Fisher's exact test; $-\log 10$ ( $\mathrm{P}$ value), the logarithmic conversion of Fisher's exact test $\mathrm{P}$ values above. 


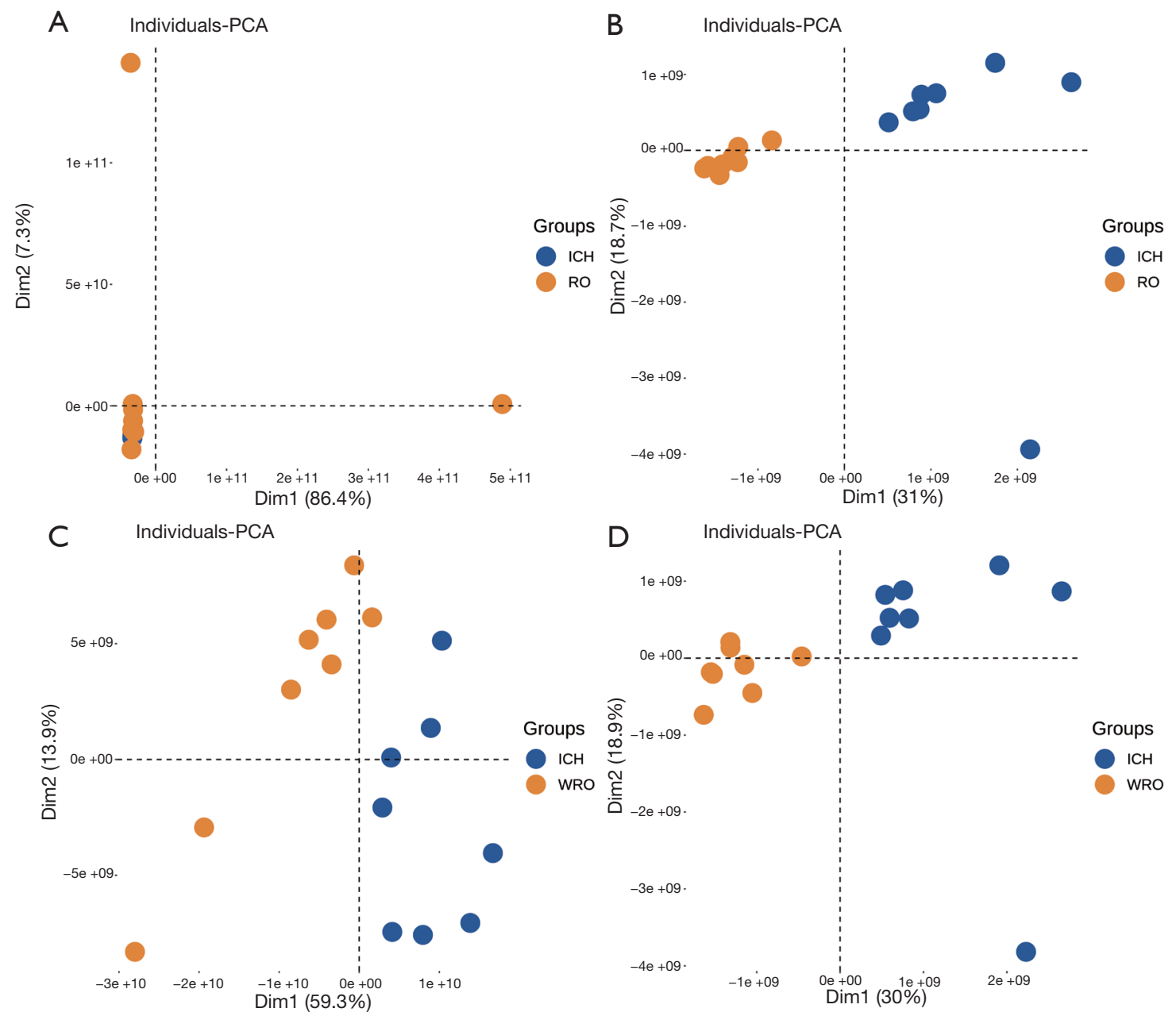

Figure 3 Principal component analysis (PCA) analysis of the intracerebral hemorrhage (ICH) model group, the raw rhubarb enema group (RO), and the wine-processed rhubarb enema group (WRO) in positive and negative ion mode. (A) PCA analysis of ICH and RO in positive ion mode; (B) PCA analysis of ICH and RO in negative ion mode; (C) PCA analysis of ICH and WRO in positive ion mode; (D) PCA analysis of ICH and WRO in negative ion mode.

\section{Partial least squares-discriminative analysis (PLS-DA) analysis}

PLS-DA, under the mode of positive and negative ions, also achieved better separation in the treatment groups with obvious differences, as shown in Figure 4. Among them, $\mathrm{R} 2 \mathrm{X}, \mathrm{R} 2 \mathrm{Y}$, and Q2 in ICH and RO positive ion mode were 0.398, 0.999, and 0.959, respectively. R2X, R2Y, and Q2 in $\mathrm{ICH}$ and RO negative ion mode were $0.373,0.994$, and 0.926, respectively. R2X, R2Y, and Q2 in ICH and WRO positive ion mode were $0.381,0.997$ and 0.949 , respectively. R2X, R2Y, and Q2 in ICH and WRO negative ion mode were $0.362,0.994$, and 0.945 , respectively. There was no fitting phenomenon in this model.

\section{Comparative analysis of the effects of RO and WRO on} the metabolism of rats with $\mathrm{ICH}$

This study compared the differences in endogenous metabolites between the RO vs. ICH groups and the WRO $v s$. ICH groups. The Venn diagram is shown in Figure 5. There were 38 identical endogenous metabolites in $\mathrm{RO} v s$. ICH and WRO vs. ICH, among which, there were 4 upregulated differential endogenous metabolites (VIP $>1$ ), including inosinic acid, 8, 9-dihetre, 3-hydroxytetradecanoic acid, and galactitol. There were 34 down-regulated differential endogenous metabolites (VIP $>1$ ), such as glutamine, 5-hydroxyindoleacetic acid, alanylvaline, 1-methylhistamine, phosphatidylinositol hemolysin, 
A

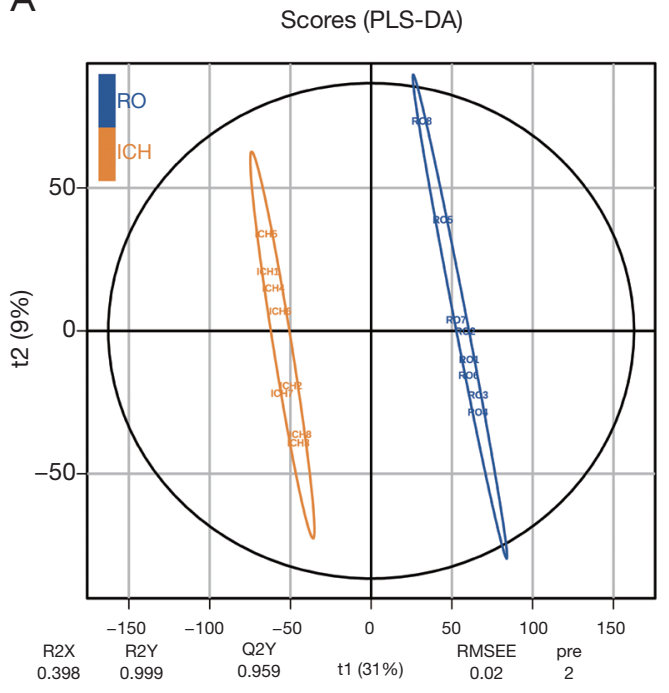

C

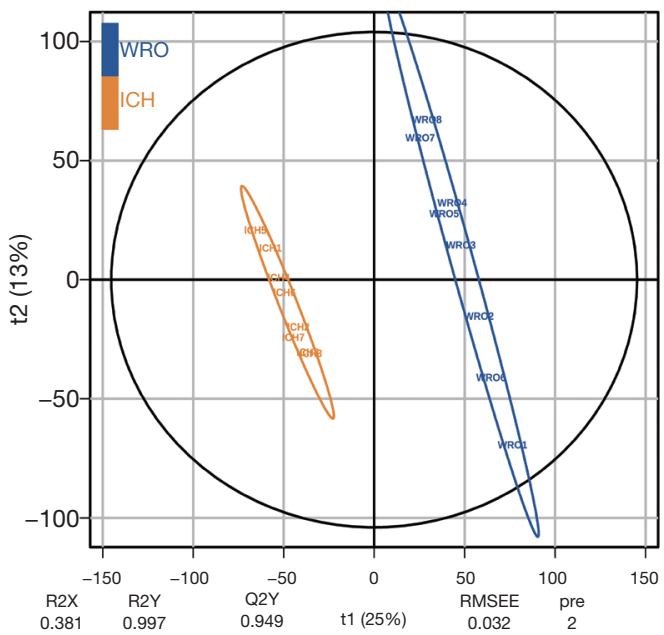

B

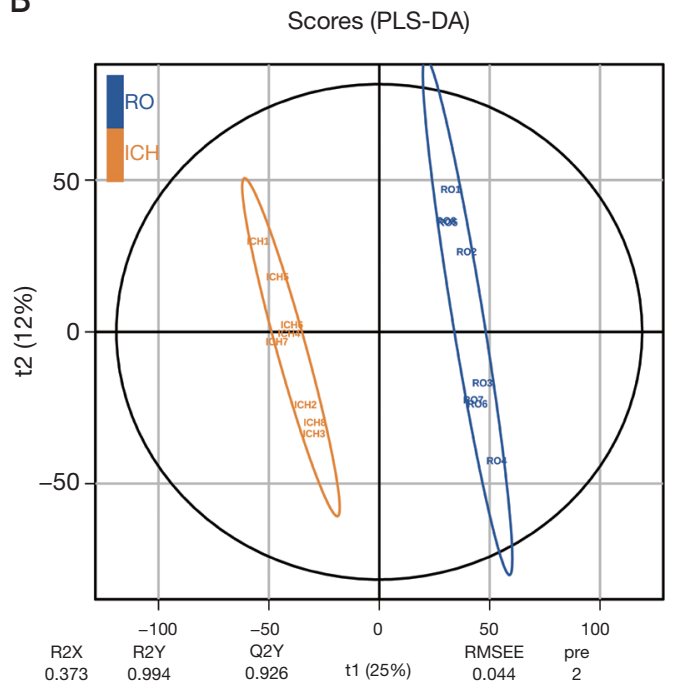

D

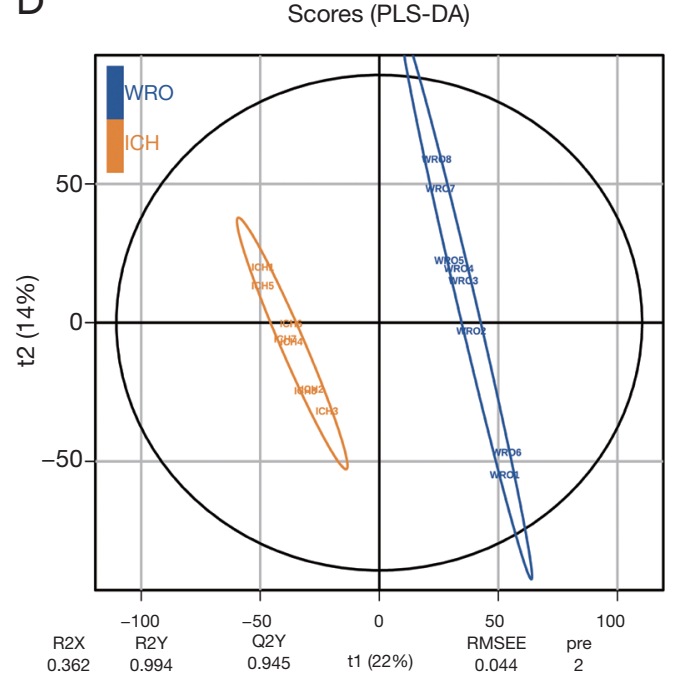

Figure 4 Partial least squares-discriminant analysis (PLS-DA) of the intracerebral hemorrhage (ICH) model group, the raw rhubarb enema group (RO), and the wine-processed rhubarb enema group (WRO) in positive and negative ion mode. (A) PLS-DA analysis of ICH and RO in positive ion mode; (B) PLS-DA analysis of ICH and RO in negative ion mode; (C) PLS-DA analysis of ICH and WRO in positive ion mode; (D) PLS-DA analysis of ICH and WRO in negative ion mode.

$\gamma$-glutamyltyrosine, taurine, and Xanthopterin. There were 23 unique differential endogenous metabolites in $\mathrm{ICH}$, including 7 up-regulated differential endogenous metabolites (VIP >1) such as GMP, UMP, n6-acetyl-1-lysine, (s)-(-)-2-hydroxy-isohexanoic acid, s-d-acyl GSH, guanine, and benzophenone. There were also 16 down-regulated differential endogenous metabolites (VIP $>1$ ), such as 7-ketocoholesterol, nucleoside, n-a-acetyl-1-arginine, GDP, sarcosine, adipic acid, inosine, and phosphatidylinositol hemolysin. There were 11 different endogenous metabolites specific to ICH, among which 3 were upregulated endogenous metabolites $(\mathrm{VIP}>1)$, including palmitoyl mercaptan, DEHP, and 6-phosphogluconic acid. There were also 8 down-regulated endogenous metabolites (VIP >1), including 3-hydroxydecanoic acid, L-tryptophan, threonine, betaine, n-acetyl-l-phenylalanine, dihydroxyacetone phosphate, galactosylsphingosine, and adenosine ribose phosphate. 

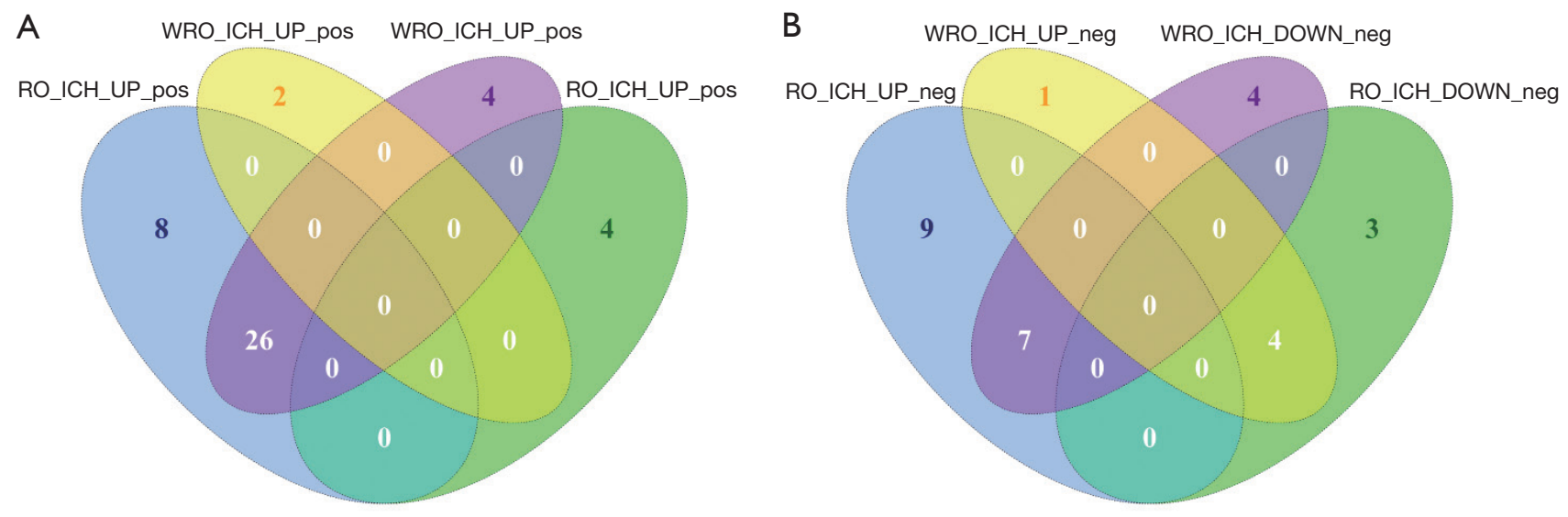

Figure 5 Comparative analysis of endogenous metabolites between the raw rhubarb enema group (RO) group $v s$. the ICH model group, and the wine-processed rhubarb enema group (WRO) vs. the ICH model group in positive and negative ion mode. (A) Positive ion mode; (B) negative ion mode.

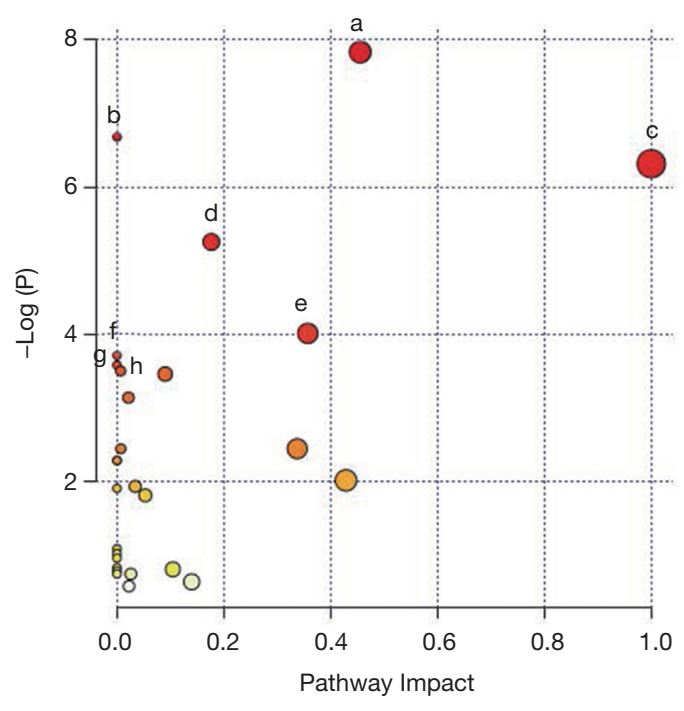

Figure 6 The common differences between raw rhubarb enema (RO) and wine-processed rhubarb enema (WRO) treatment expressed the Kyoto Encyclopedia of Genes and Genomes (KEGG) pathway annotation of metabolites. a, $\beta$-alanine metabolism; b, aminoacyl tRNA biosynthesis; c, purine metabolism; d, phenylalanine metabolism; e, histidine metabolism; f, pyrimidine metabolism; g, niacin ester and niacinamide metabolism; h, pantothenic acid and CoA biosynthesis.

\section{Metabolic co-mechanism of RO and WRO treatment in ICH rats}

In order to further analyze the effects of endogenous metabolites shared by RO vs. ICH and WRO vs. ICH treatment on rats with $\mathrm{ICH}$, the related metabolic pathways of these different endogenous metabolites were analyzed by KEGG pathway data, and the pathway with $\mathrm{P}<0.05$ was taken as the related important pathway, as shown in Figure 6. It was found that with RO and WRO treatment, the main metabolic pathways that were altered included $\beta$-alanine metabolism, aminoacyl tRNA biosynthesis, purine metabolism, phenylalanine metabolism, histidine metabolism, pyrimidine metabolism, niacin ester and niacinamide metabolism, and pantothenic acid and CoA biosynthesis.

\section{Analysis of specific mechanisms of the metabolome in rats with ICH treated with RO}

Through KEGG pathway data analysis, the related metabolic pathways of endogenous metabolites related to differential expression were analyzed, and the pathway of $\mathrm{P}<0.05$ was taken as the related important pathway, as shown in Figure 7. Compared with the WRO group, purine metabolism and riboflavin metabolism were the main metabolic pathways in the $\mathrm{RO}$ group.

\section{Analysis of specific mechanisms of the metabolome in rats with ICH treated with WRO}

Through KEGG pathway data analysis, the related metabolic pathways of endogenous metabolites related to differential expression were analyzed, and the pathway of $\mathrm{P}<0.05$ was taken as the related important pathway, as shown in Figure 8. Compared with the RO group, glycine, serine, and threonine, and the biosynthesis of aminoacyl tRNA were the main metabolic pathways in the WRO group. 


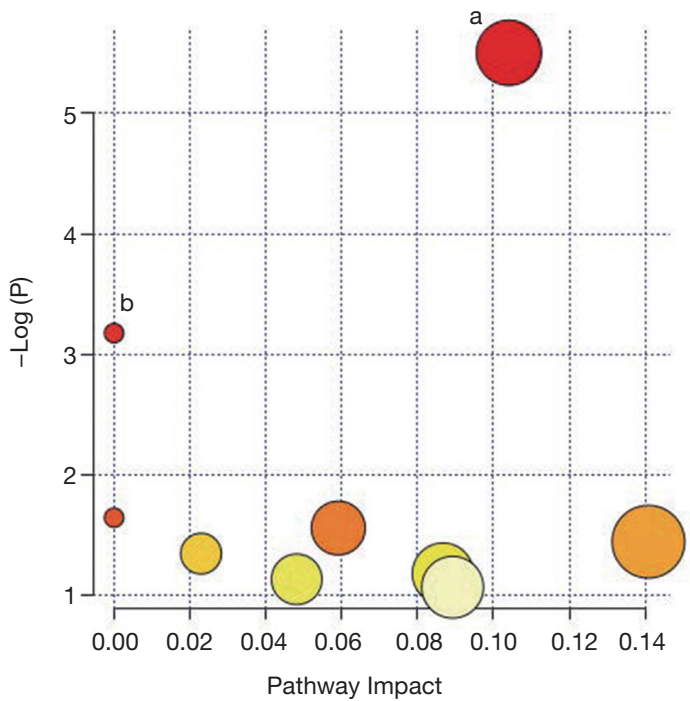

Figure 7 Pathway annotation of Kyoto Encyclopedia of Genes and Genomes (KEGG) for specific differentially expressed metabolites treated with raw rhubarb enema (RO). a, purine metabolism; b, riboflavin metabolism.

\section{Discussion}

Effect of raw rhubarb and wine-processed rbubarb on the protein expression profiles of rats with ICH

In the differential protein analysis of the $\mathrm{ICH}, \mathrm{RO}$, and WRO groups, 309 and 210 kinds of differential proteins related to the raw and wine-processed rhubarb were found, compared with the ICH model group. Enrichment analysis showed that both treatments affected protein expression on macrophages, lysosomes, and cytoskeletons, and regulated the biological processes of postsynaptic specialization and phospholipid metabolism through the molecular function of cadherin binding and RNA binding. These proteins affect the pathway of amino acid metabolism and signal transduction.

Compared with raw rhubarb, wine-processed rhubarb had a better effect on the expression of Acp6, Cacna2d2, Commd 3 and other proteins, which effectively played a role in the repair of ICH. Meanwhile, the expression of Aldh1l1, Ciapin1, Ncstn and other related proteins in raw rhubarb had an excessive effect on the recovery of cerebral hemorrhage injury. This might also have affected Gltp, Hnrnpc, Khsrp, Loc100911677, Mtmr7 and other proteins. Raw rhubarb and wine-processed rhubarb had the same performance, indicating that both rhubarb treatments have the ability to repair cerebral hemorrhage injury.

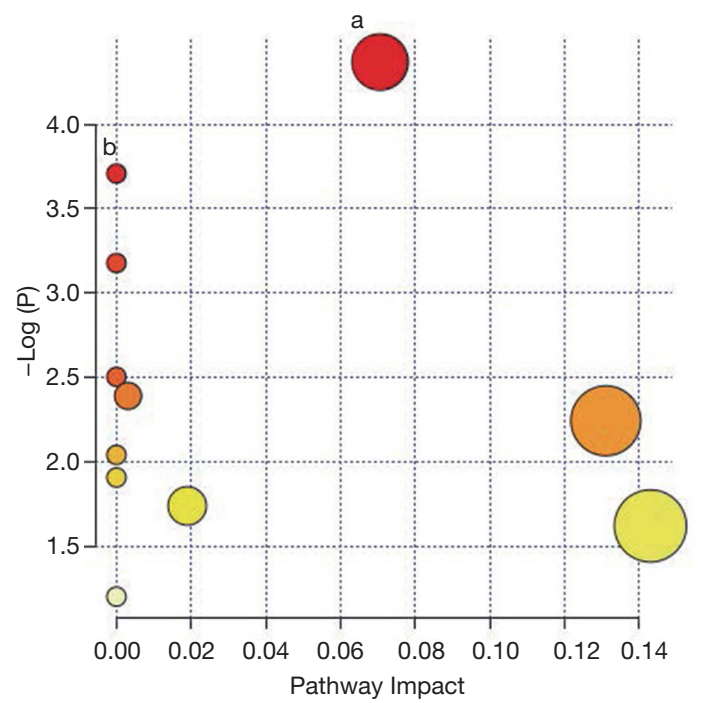

Figure 8 Pathway annotation of Kyoto Encyclopedia of Genes and Genomes (KEGG) for specific differentially expressed metabolites treated with wine-processed rhubarb enema (WRO). a, biosynthesis of aminoacyl tRNA; b, metabolism of glycine, serine, and threonine.

After hemorrhage, due to massive platelet coagulation, leukocyte infiltration, and other processes, inflammation of the nervous system leads to brain edema and secondary apoptosis of nerve cells (9). Proteomics analysis of the 2 kinds of rhubarb treatment in this study showed that leukocyte infiltration and platelet coagulation-related protein expression decreased in the surrounding tissue of the hematoma. The AMPK pathway can activate autophagy of the nervous system, reduce oxidative stress levels in brain tissue, protect injured nerve cells, improve energy supply, and thus reduce apoptosis of nerve cells (10). Both rhubarb treatments effectively reduced oxidative stress responses and inhibited neuronal apoptosis through the AMPK pathway, while both of the rhubarb treatment also activated the PPAR pathway and increased the activation level of the AMPK pathway.

In summary, it was found that oxidative stress activated inflammatory cytokines after ICH, and up-regulated platelet activating factors induced the interaction of platelets, leukocytes, and vascular endothelial cells to block microvessels, leading to brain edema and neuronal damage. Both rhubarb treatments protected the blood-brain barrier by anti-inflammatory and anti-oxidative stress responses, protected the injured nerve cells during the process of ICH, reduced excitatory amino acids, and restored brain tissue 
homeostasis. Compared with raw rhubarb, wine-processed rhubarb had a better therapeutic effect on ICH.

\section{Effects of ICH on metabolism in rats}

Previous studies have shown that high levels of phenylalanine have toxic effects on nerve cells, activating the NMDR$\mathrm{Ca}^{2+}$-CaMK II-RAS-p38 axis, causing abnormal neuronal processes (11). In the environment of high phenylalanine, neurotransmitters are released abnormally, and the excitatory neurotransmitter glutamate may be involved in neuronal damage caused by high phenylalanine (12). It was found that L-lysine can reduce damage to neurons, promote the absorption and axon extension of neurons, and improve the activity of neurons (13). Results have also shown that the levels of alanine decreased, which suggested that immunity was low after ICH, and the glucose alanine cycle was blocked (14).

Recent studies have shown that impaired energy metabolism around the intracerebral hematoma is an important cause of secondary injury in ICH. After ICH, the supply of factors needed for energy metabolism is interrupted, which leads to impaired energy metabolism and energy consumption, and leads to a cascade reaction after ICH injury. The increase content of serine, one of the glycogenic and ketogenic amino acids, is associated with energy starvation and reduction of glycolysis in the ICH brain tissue (15), glucose utilization is reduced, utilization of glycogenic amino acids increases, and gluconeogenesis increases (16). After ICH, abnormal energy metabolism and gluconeogenesis in brain tissue affect the metabolism of fatty acids and the stability of the TCA cycle, which eventually leads to a decrease in immune function and the toxicity of excitatory amino acids to nerve cells.

After the occurrence of ICH, the metabolites in the brain tissue of rats with ICH changed significantly, and mainly manifested as energy metabolism disorder. The stress changes caused by hemorrhage, such as energy deficiency, altered neurotransmission, and metabolic abnormality, resulted in the death of nerve cells after the occurrence of ICH. The study of metabolites after ICH is therefore helpful to further clarify the molecular mechanisms of brain tissue damage after ICH.

\section{Effects of raw rhubarb and wine-processed rbubarb on metabolism in rats with ICH}

In this study, metabolomics was used to analyze endogenous metabolites in the rat model of ICH, and to analyze the differences in endogenous metabolites before and after rhubarb wine roasting, and a considerable number of different endogenous metabolites were obtained. This analysis can be regarded as a potential biomarker or treatment pathway after the occurrence of disease, and provides a basis for understanding the mechanism of disease action and verifying the drug mode of action.

PCA and PLS-DA were used to analyze the therapeutic effect of rhubarb on ICH in rats. It was found that the endogenous metabolites of RO and WRO were distinct from ICH, indicating that both rhubarb treatments could effectively protect the physiological and metabolic status of rats after ICH. The comparative analysis of each group showed that some of the metabolites from rhubarb treatment still coincided with the ICH model group, such as PCA analysis in the positive ion mode, while wineprocessed rhubarb did not coincide with the ICH model group in various ion modes, indicating that wine-processed rhubarb had a better protective effect on ICH compared with raw rhubarb.

Compared with ICH group, 53 different endogenous metabolites were found in the raw rhubarb group, while 58 were found in the wine-processed group, including inosine and 8,9-dihetre, 3-hydroxytetradecanoic acid, galactitol, glutamine, 5-hydroxyindoleacetic acid, alanylvaline, 1-methylhistamine, phosphatidylinositol hemolysin, $\gamma$-glutamyltyrosine, taurine, and xanthine. In the pathway analysis, we found that these different endogenous metabolites mainly affect the biosynthesis of tyrosine and tryptophan, $\beta$-alanine, phenylalanine, purine, histidine, pantothenic acid and CoA, pyrimidine, aminoacyl tRNA, arginine, and niacin ester and niacinamide. In addition, RO treatment can affect purine metabolism, GSH metabolism, and riboflavin metabolism pathways, while WRO treatment can affect glycine, serine, and threonine metabolism, aminoacyl tRNA biosynthesis, valine, leucine, and isoleucine biosynthesis pathway.

In the analysis of the differential metabolites, it was found that compared with raw rhubarb, wine-processed rhubarb had a better effect on the levels of $\gamma$-glutamyltyrosine, glutamine, and other metabolites, and played an effective role in the repair of ICH. At the same time, the content of $\beta$-alanine, cytidine, xanthine, and other related metabolites was significantly changed after treated by both raw rhubarb and wine-processed rhubarb, which may affect the recovery of ICH. Additionally, 5-aminovaleric acid, alanylvaline, aspartame, benzaldehyde, and other metabolites were 
regulated after raw rhubarb and wine-processed rhubarb treatment, suggesting that both rhubarb treatments have the ability to restore ICH injury.

Insulin can promote cell absorption and use of branched chain amino acids, reducing blood glucose levels (17). Studies have shown that phenylalanine can promote insulin secretion, which may be related to the increased nutritional supply of phenylalanine (18). On the one hand, high insulin level destroys the balance of amino acid metabolism in tissues, reduces the concentration of branched chain amino acids in blood, and increases the concentration of aromatic amino acids. Aromatic amino acids (such as phenylalanine) use the same carrier as branched amino acids to cross the blood-brain barrier and competitively inhibit branched amino acids from entering brain tissue (19), resulting in the reduction of branched amino acid metabolism and insufficient energy supply. On the other hand, hypoglycemia can lead to insufficient energy supply of brain cells (20). The combined effects of these 2 processes can lead to brain damage and increase damage in ICH. Both RO and WRO treatment reduced phenylalanine metabolism, demonstrating a neuroprotective effect.

Uridine nucleotide is the main source of extracellular uridine triphosphate (UTP) and uridine diphosphate (UDP). When oxygen levels are normal, uridine nucleotide is converted into UTP, but when blood oxygen levels are low due to the lack of ATP, the conversion efficiency of this uridine nucleotide UTP loop is reduced, and will be decomposed into uridine (21). Consistent with the state of energy deficiency, The increase in uracil content enhances the metabolic capacity of in the brain tissue of rats with ICH. After RO and WRO treatment, the content of uracil in the body of rats decreased, and the level of metabolites of uracil decreased, indicating that the impairment in energy metabolism was alleviated to a certain extent, and the energy supply had been restored.

Serine is a kind of nonessential amino acid, which plays an important role in many physiological activities. Phosphoserine, the main component of phospholipids, is an endogenous metabolite with physiological activity produced by the phosphorylation of serine hydroxyl. Phosphorylation of serine, threonine, and tyrosine can regulate the expression rate of insulin receptors. During WRO treatment, the serine levels and phosphorylation levels of brain tissue increased, which ensured glucose transport ability in brain tissue. WRO treatment provided more energy for brain tissue through such mechanisms, which might have been the reason why WRO treatment had a better protective effect on ICH in rats.

GSH is composed of glycine, cysteine, and glutamic acid. It is widely found in blood and plays an important role in the organism. GSH has 2 forms of oxidation and reduction (22). It is the cofactor of most enzyme reactions. It can be used as an antioxidant to scavenge oxygen free radicals and protect biological macromolecules (23). GSH is involved in the process of the tricarboxylic acid cycle and glucose metabolism $(24,25)$. Its antioxidant effect can resist the oxidative stress caused by external injury (23). Oxidative stress is one of the main phenotypes of brain injury (26). The decrease in antioxidant levels is mainly caused by the decrease of GSH synthesis precursors cysteine and glycine (27). Treatment with wine-processed rhubarb increased the metabolism of glycine, while treatment with raw rhubarb increased the intensity of GSH metabolism. Therefore, both rhubarb treatments improved the level of GSH metabolism. This exogenous rhubarb treatment can improve the metabolism level of riboflavin, which is one of the essential vitamins for the human body. Many studies have found that riboflavin is closely related to human cardiovascular and cerebrovascular diseases, cancer, and other diseases (28). Riboflavin is involved in the metabolism of folic acid and homocysteine in brain injury, which indirectly inhibits the occurrence of brain injury (29,30). Riboflavin can improve the level of antioxidation. Rhubarb treatment specifically increased the expression of riboflavin, which can also play a better role in antioxidant defenses.

In conclusion, both of the 2 rhubarb treatments improved energy metabolism and inhibited the oxidative stress caused by ICH. By increasing the metabolism of $\beta$-alanine, the energy supply was increased, and the metabolism of glutamine was decreased. The results suggest that both rhubarb treatments can therefore reduce the oxidative stress response caused by hemorrhage, reduce the production of reactive oxygen species, avoid oxidative stress injury, and restore the redox balance of brain tissue to protect damaged nerve cells.

\section{Conclusions}

In this study, we performed proteomics and metabolomics on the brain tissue of ICH rats treated with raw rhubarb and wine-processed rhubarb. Both rhubarb treatments alleviated oxidative stress responses. Pathway enrichment showed that compared to raw rhubarb treated ICH rats, wine-processed rhubarb treatment might regulate amino acid metabolism. Analysis of the differential metabolites of raw rhubarb 
and wine-processed rhubarb treated rats showed that both rhubarb treatments suppressed phenylalanine metabolism, demonstrating a neuroprotective effect. Wine-processed rhubarb was implicated in the metabolism of glutamine, which might be associated with its better therapeutic effect in ICH. Our study therefore provides a basis for further research on the mechanism of rhubarb treatment from different processing technologies, and promotes the application of rhubarb as a therapeutic approach to ICH.

\section{Acknowledgments}

Funding: This study was supported by the National Natural Science Foundation of China (Grant No.81573919). The work was also supported by the Project of Scientific Research on Traditional Chinese Medicine in Henan Province (No.20-21ZYZD08, No. 2019ZY2037 and No. 2018ZY2053).

\section{Footnote}

Reporting Checklist: The authors have completed the ARRIVE reporting checklist. Available at http://dx.doi. org/10.21037/atm-20-7831

Data Sharing Statement: Available at http://dx.doi. org/10.21037/atm-20-7831

Conflicts of Interest: All authors have completed the ICMJE uniform disclosure form (available at http://dx.doi. org/10.21037/atm-20-7831). The authors have no conflicts of interest to declare.

Ethical Statement: The authors are accountable for all aspects of the work in ensuring that questions related to the accuracy or integrity of any part of the work are appropriately investigated and resolved. Experiments in our research were performed under a project license granted by the Institutional Animal Care and Use committee of Henan Province Hospital of TCM (Second Affiliated Hospital of Henan University of Traditional Chinese Medicine), in compliance with national guidelines for the care and use of animals.

Open Access Statement: This is an Open Access article distributed in accordance with the Creative Commons Attribution-NonCommercial-NoDerivs 4.0 International License (CC BY-NC-ND 4.0), which permits the non- commercial replication and distribution of the article with the strict proviso that no changes or edits are made and the original work is properly cited (including links to both the formal publication through the relevant DOI and the license). See: https://creativecommons.org/licenses/by-nc-nd/4.0/.

\section{References}

1. Ikram MA, Wieberdink RG, Koudstaal PJ. International epidemiology of intracerebral hemorrhage. Curr Atheroscler Rep 2012;14:300-6.

2. Hemphill JC 3rd, Greenberg SM, Anderson CS, et al. Guidelines for the Management of Spontaneous Intracerebral Hemorrhage: A Guideline for Healthcare Professionals From the American Heart Association/ American Stroke Association. Stroke 2015;46:2032-60.

3. Wilkinson DA, Keep RF, Hua Y, et al. Hematoma clearance as a therapeutic target in intracerebral hemorrhage: From macro to micro. J Cereb Blood Flow Metab 2018;38:741-5.

4. Schlunk F, Greenberg SM. The Pathophysiology of Intracerebral Hemorrhage Formation and Expansion. Transl Stroke Res 2015;6:257-63.

5. Wang Y, Peng F, Xie G, et al. Rhubarb attenuates bloodbrain barrier disruption via increased zonula occludens-1 expression in a rat model of intracerebral hemorrhage. Exp Ther Med 2016;12:250-6.

6. Wu Y, Peng XQ, Jiang XY, et al. Effects of wine processed Rheum palmatum on tissue distribution of aloe-emodin, rhein and emodin in rats. Zhongguo Zhong Yao Za Zhi 2017;42:1603-8.

7. Deng S, Feng S, Wang W, et al. Biomarker and Drug Target Discovery Using Quantitative Proteomics PostIntracerebral Hemorrhage Stroke in the Rat Brain. J Mol Neurosci 2018;66:639-48.

8. Andrejeva G, Rathmell JC. Similarities and Distinctions of Cancer and Immune Metabolism in Inflammation and Tumors. Cell Metab 2017;26:49-70.

9. Zhang X, Li H, Hu S, et al. Brain edema after intracerebral hemorrhage in rats: the role of inflammation. Neurol India 2006;54:402-7.

10. Shi H, Wang J, Wang J, et al. IL-17A induces autophagy and promotes microglial neuroinflammation through ATG5 and ATG7 in intracerebral hemorrhage. J Neuroimmunol 2018;323:143-51.

11. Lapchak PA, Chapman DF, Zivin JA. Metalloproteinase inhibition reduces thrombolytic (tissue plasminogen activator)-induced hemorrhage after thromboembolic 
stroke. Stroke 2000;31:3034-40.

12. Zhang X, Li Y, Liang $Y$, et al. Distinguishing Intracerebral Hemorrhage from Acute Cerebral Infarction through Metabolomics. Rev Invest Clin 2017;69:319-28.

13. Droz B, Koenig HL, Biamberardino LD, et al. Axonal migration of protein and glycoprotein to nerve endings. I. Radioautographic analysis of the renewal of protein in nerve endings of chicken ciliary ganglion after intracerebral injection of (3H)lysine. Brain Res 1973;60:93-127.

14. Seo GH, Oh A, Kim EN, et al. Identification of extremely rare mitochondrial disorders by whole exome sequencing. J Hum Genet 2019;64:1117-25.

15. Paoli A, Bianco A, Damiani E, et al. Ketogenic diet in neuromuscular and neurodegenerative diseases. Biomed Res Int 2014;2014:474296.

16. Zinnanti WJ, Lazovic J, Housman C, et al. Mechanism of metabolic stroke and spontaneous cerebral hemorrhage in glutaric aciduria type I. Acta Neuropathol Commun 2014;2:13.

17. Sharma AK, Taneja G, Kumar A, et al. Insulin analogs: Glimpse on contemporary facts and future prospective. Life Sci 2019;219:90-9.

18. Kun S, Molnár GA, Sélley E, et al. Insulin Therapy of Nondiabetic Septic Patients Is Predicted by para-Tyrosine/ Phenylalanine Ratio and by Hydroxyl Radical-Derived Products of Phenylalanine. Oxid Med Cell Longev 2015;2015:839748.

19. Germanó A, d'Avella D, Cicciarello R, et al. Bloodbrain barrier permeability changes after experimental subarachnoid hemorrhage. Neurosurgery 1992;30:882-6.

20. Yang GY, Betz AL, Chenevert TL, et al. Experimental intracerebral hemorrhage: relationship between brain edema, blood flow, and blood-brain barrier permeability in rats. J Neurosurg 1994;81:93-102.

Cite this article as: Wang K, Guan D, Zhao X, Qiao D, Yang Y, Cui Y. Proteomics and metabolomics of raw rhubarb and wineprocessed rhubarb in the treatment of rats with intracerebral hemorrhage. Ann Transl Med 2020;8(24):1670. doi: 10.21037/ atm-20-7831
21. Zheng J, Shi L, Liang F, et al. Sirt3 Ameliorates Oxidative Stress and Mitochondrial Dysfunction After Intracerebral Hemorrhage in Diabetic Rats. Front Neurosci 2018;12:414.

22. Anjum I, Jaffery SS, Fayyaz M, et al. Sugar Beverages and Dietary Sodas Impact on Brain Health: A Mini Literature Review. Cureus 2018;10:e2756.

23. Gebicki JM, Nauser T, Domazou A, et al. Reduction of protein radicals by GSH and ascorbate: potential biological significance. Amino Acids 2010;39:1131-7.

24. Huang Q, Tan Y, Yin P, et al. Metabolic characterization of hepatocellular carcinoma using nontargeted tissue metabolomics. Cancer Res 2013;73:4992-5002.

25. Vaillancourt F, Fahmi H, Shi Q, et al. 4-Hydroxynonenal induces apoptosis in human osteoarthritic chondrocytes: the protective role of glutathione-S-transferase. Arthritis Res Ther 2008;10:R107.

26. Finkel T, Holbrook NJ. Oxidants, oxidative stress and the biology of ageing. Nature 2000;408:239-47.

27. Koskenkorva-Frank TS, Weiss G, Koppenol WH, et al. The complex interplay of iron metabolism, reactive oxygen species, and reactive nitrogen species: insights into the potential of various iron therapies to induce oxidative and nitrosative stress. Free Radic Biol Med 2013;65:1174-94.

28. Gariballa S, Ullegaddi R. Riboflavin status in acute ischaemic stroke. Eur J Clin Nutr 2007;61:1237-40.

29. Ward M, Wilson CP, Strain JJ, et al. B-vitamins, methylenetetrahydrofolate reductase (MTHFR) and hypertension. Int J Vitam Nutr Res 2011;81:240-4.

30. Abdollahi A, Omranipour R. Is Increase of Homocysteine, Anti-Cardiolipin, Anti-Phospholipid Antibodies associated with Breast Tumors? Acta Med Iran 2015;53:681-5.

(English Language Editor: C. Betlazar-Maseh) 

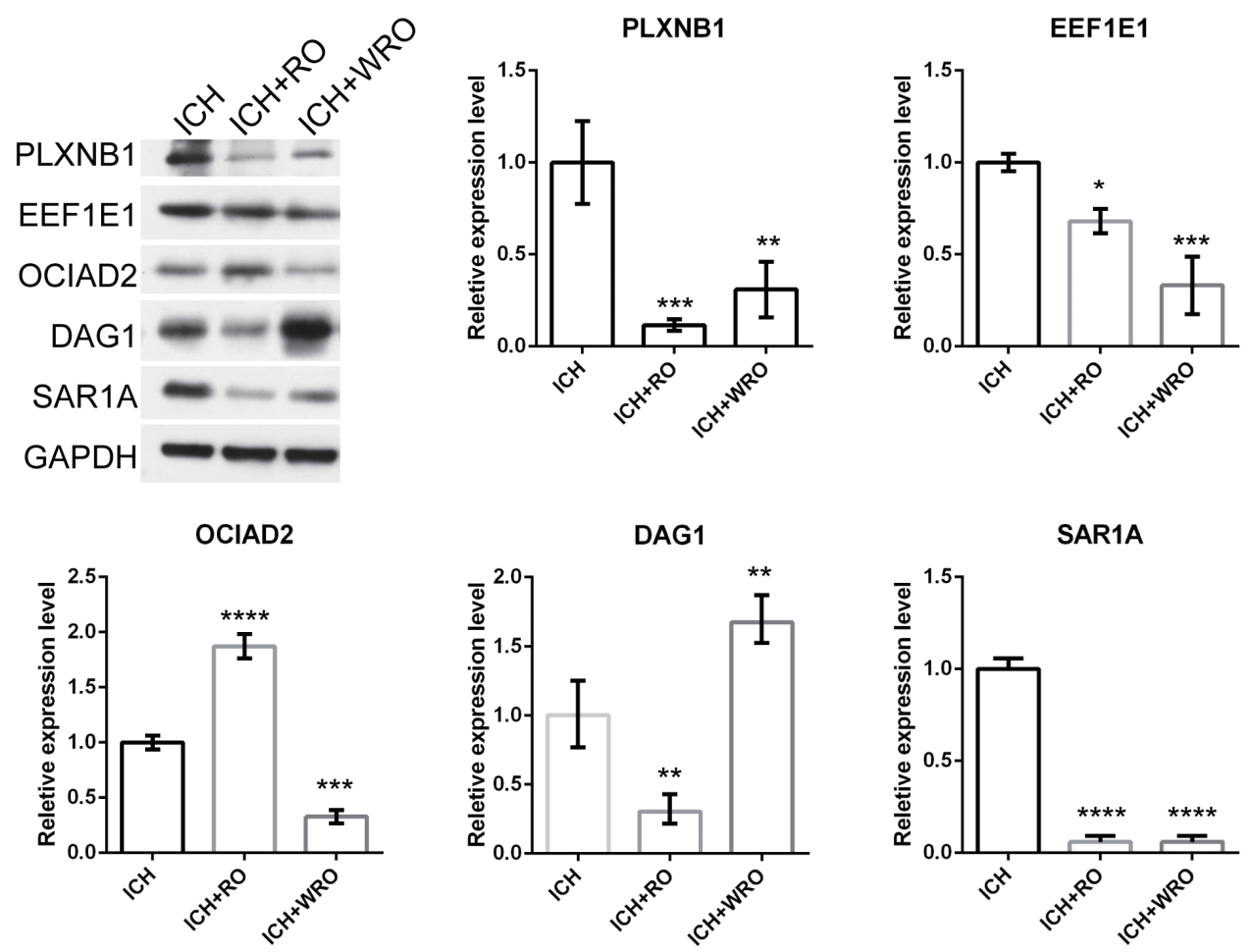

Figure S1 Verification of the expression of differential expressed proteins. Western blotting was performed to detect the expression of PLXNB1, EEF1E1, OCIAD2, DAG1, and SAR1A among ICH group, ICH + RO group, and ICH + WRO group. ${ }^{*} \mathrm{P}<0.05,{ }^{*} \mathrm{P}<0.01$, ${ }^{* * *} \mathrm{P}<0.001,{ }^{* * * *} \mathrm{P}<0.0001$. 